\title{
transCryptFS: A Trusted and Secure File System
}

\author{
Rajat Moona \\ Department of Computer Science and Engineering, IIT Kanpur \\ moona@iitk.ac.in
}

\begin{abstract}
For the reasons of flexibility and availability, the data may be stored in the cloud. However security of sensitive data and the reliability of access of data are two major issues that become immediate concern. Trust on the services for the secure upkeep of data, its regular maintenance and mechanisms for seamless access are other issues related to such organization of data.

In this talk, we present an encrypting file system based on GNU/Linux based servers which can be accessed through standard protocols such as NFS over unsecured networks. At the same time, the data stored in the servers is always in encrypted form. With only a suitable key management protocol and access control mechanisms, it is ensured that data is made available only to right entities. In the trusted domain for the files, even the system administrators are not included which makes it highly robust yet providing mechanisms for routine administrative jobs such as backup, restoration, duplication and other similar functions on data.

We also present a few more applications for this same file system, such as the case where data on a portable device such as laptop can be protected against loss of information in case of thefts etc.
\end{abstract}

